\title{
Las lenguas paleoeuropeas. Una aproximación historiográfica a la edición de sus textos
}

\section{Palaeoeuropean languages. A historiographical approach to the edition of their texts}

Resumen: La edición de los textos pertenecientes a las lenguas europeas antiguas (paleoeuropeas) se ha visto condicionada por el avance experimentado en otras disciplinas que guardan gran relación con ella, así como por los progresos tecnológicos más recientes. En este capítulo se describe la historia de la aproximación científica a los textos de las antiguas lenguas de Europa, que parte del análisis más puramente textual y llega hasta la equilibrada interacción entre todos los campos. Este recorrido historiográfico se ha estructurado en tres etapas: desde los inicios en los siglos XVIII y XIX hasta la mitad del XX; la segunda mitad del XX y, por último, los comienzos del siglo XXI. Finalmente se contextualiza el aporte de la red AELAW y se sugieren algunas prospectivas de estudio.

Palabras clave: Historia de la Epigrafía. Lenguas paleoeuropeas. Escrituras paleoeuropeas, Corpora epigráficos. Historiografía. Humanidades Digitales.

Abstract: The edition of texts written in the ancient European languages (Palaeoeuropean) has been substantially conditioned by the progress of other disciplines that are narrowly linked to it and by recent technological developments. This chapter describes the history of the scientific approach to Palaeoeuropean texts that started as a purely textual analysis and is currently the result of a balanced interaction of every field. This historiographic panorama is structured in three phases: from its first steps in the $18-19^{\text {th }}$ centuries until the middle of the $20^{\text {th }}$, the second half of the $20^{\text {th }}$ century and, last, the first decades of the $21^{\text {st }}$. Finally, the contribution of the AELAW network is contextualized and some potential lines of research are suggested.

Key words: History of Epigraphy. Palaeoeuropean Languages. Palaeoeuropean Writings. Epigraphic corpora. Historiography. Digital Humanities.

Recepción: 21.11.2019 | Aceptación: 22.03.2020

Financiación: Este artículo se enmarca en los proyectos de investigación FFI2015-63981C3-1-P del Ministerio de Economía, Industria y Competitividad, y en el marco del Grupo Consolidado del Gobierno Vasco - Eusko Jaurlaritza GIC12/92-IT698-13; y su redacción ha sido posible gracias a los contratos Juan de la Cierva - Incorporación IJCI-201627589 y Ramón y Cajal RYC2018-024089-I (Fondo Social Europeo - Agencia Estatal de Investigación). 


\section{"Instead of attempting to obtain new (highly hypothetical, and almost useless) translations of untranslatable words, scholars began \\ to employ what we do understand in (...) inscriptions to reconstruct social and political histories of (...) cities"}

Benelli 2017, 103.

\section{Introducción}

1.1. Desde los comienzos de la investigación sobre las antiguas lenguas y escrituras de Europa, para los que nos podemos remontar al siglo XVIII en algunos conjuntos epigráficos, ${ }^{1}$ se puede percibir la fascinación que suscitaban aquellas inscripciones, por entonces enigmáticas e indescifrables. Estos primeros trabajos están trufados de elucubraciones sobre mundos antiguos y civilizaciones primigenias sobre las que posteriormente se sustentaron postulados nacionalistas con el fin de encontrar una base cultural y lingüística en la que apoyarse. ${ }^{2}$

Hasta llegar a los trabajos actuales, resultado de investigaciones de carácter más positivista e interdisciplinar, la disciplina ha pasado por varias fases, acordes con su tiempo y con los avances científicos que se han ido produciendo. Proponemos en este artículo un sucinto ${ }^{3}$ tránsito por dicho camino historiográfico, deteniéndonos en algunos de los principales corpora epigráficos que contienen textos escritos en las antiguas lenguas y escrituras de Europa. Ubicaremos el foco de atención especialmente en la edición de los textos y

1 Nos referimos fundamentalmente al conjunto etrusco, el más precoz en recibir atención. Estas primeras obras fueron resultado de la llamada "Etruscheria", que podría traducirse como "etruscomanía" del siglo XVIII, y algunos de sus principales promotores fueron S. Maffei o A. F. Gori. Sobre ella, Della Fina 2017, 57-61 y Benelli 2017, 95-97.

2 Es lo que ocurrió, por ejemplo, con el Corpus Inscriptionum Italicarum de A. Fabretti (Fabretti 1867, ver Benelli 2017, 95).

3 El presente trabajo ha de entenderse como una introducción historiográfica al estado de la cuestión del estudio de las lenguas paleoeuropeas y no como un análisis exhaustivo. Por ello, en aras de la claridad expositiva y de la coherencia de nuestro discurso, hemos seleccionado las obras más representativas. El lector posiblemente eche en falta trabajos fundamentales; pero no era posible en el marco de este trabajo abordar todos los corpora y sus complementos, todas las gramáticas y las compilaciones de las casi treinta lenguas antiguas de Europa. 
en el "espíritu" con el que esta se realizó (de tipo filológico, arqueológico, epigráfico, histórico, etc.), con el fin de poner en valor el camino recorrido hasta nuestros días, en los que celebramos la proliferación de bancos de datos epigráficos online y proyectos como AELAW, en el que se apuesta claramente por la inscripción en sí como objeto de estudio donde confluyen trabajos de especialistas de diversa formación, a diferencia de otros enfoques en los que la lengua es la exclusiva protagonista, por ejemplo, y a los que solo pueden acceder investigadores versados en dicha materia.

1.2. En nuestro estado actual de conocimientos, consideramos la existencia de 28 lenguas fragmentariamente atestiguadas, directa o indirectamente, que se hablaron en el Occidente europeo antes de la difusión del latín. ${ }^{4}$ Entre todas ellas existe una gran diferencia en cuanto a sus características externas y documentación: algunas son lenguas aisladas y otras, por el contrario, se encuentran englobadas en familias bien conocidas; la mayoría tienen una documentación limitada y unas pocas, en cambio, cuentan con miles de textos; algunas se escribieron durante siglos y otras no documentan más que unas pocas atestiguaciones ocasionales.

1.3. A diferencia de las obras sobre las lenguas latina y griega, mucho mejor conocidas, los compendios sobre las Trümmersprachen ${ }^{5}$ europeas han tenido que pasar por una primera fase en la cual no llegaba a leerse con total certeza el contenido de lo que se estaba editando porque el sistema de escritura en cuestión aún no estaba descifrado con un grado de seguridad razonable. De esta primera fase, caracterizada por la abundancia de postulados hipotéticos, se pasó a un segundo estadio posterior al desciframiento de la escritura correspondiente (en los casos en los que esta era vernácula), en el que la aproximación lingüística a los textos adquirió una mayor relevancia. Esta etapa, completamente necesaria para el avance de la investigación, se ha visto sucedida por un tercer periodo en el que la edición del texto está compensada por datos de carácter arqueológico e histórico; una fase en la que no se estudia solo el texto sino también su contexto y su materialidad.

4 En el censo de $A E L A W$ (<http://aelaw.unizar.es/database/languages $>$ ) se han recontado 27 lenguas de fragmentaria atestación, a las que habría que añadir el tracio, y unos 17.000 textos (uid. infra, apartado 5).

5 Las "lenguas fragmentarias" o "lenguas de restos" (Restsprachen) son denominadas en ocasiones como "lenguas ruinosas" o "Trümmersprachen", según la terminología de Untermann 1980. Para el ámbito indoeuropeo es interesante el coloquio de la Società Italiana di Glottologia y de la Indogermanische Gesellschaft, editado por Vineis 1983, en el que destacan los trabajos de Untermann 1983 y Campanile 1983. 
Por último, la entrada en escena de las bases de datos informáticas ha permitido en años recientes un nuevo cambio en la edición de los epígrafes, con miras a gestionar mejor el almacenamiento, la consulta y las búsquedas, lo que homogeneiza enormemente las fichas epigráficas y su contenido. Estos buscadores están permitiendo avanzar a pasos agigantados en el conocimiento de las lenguas paleoeuropeas, ya que la escasez de los datos intrínsecos de cada inscripción se puede compensar en cierta medida con el fácil hallazgo de paralelos gracias al procesamiento informatizado de "big data epigráficos". El resultado, derivado de esta información de tipo comparativo, está siendo claramente satisfactorio, pues en muchos casos nos encontramos ante textos formulares y repetitivos cuya interpretación mejora de forma manifiesta al ser puestos en su contexto documental. Una explicación más amplia de las cuatro fases que se acaban de enumerar sigue en las próximas líneas.

\section{Investigando "a ciegas". El estudio de las lenguas y las escrituras paleoeuropeas hasta la primera mitad del siglo $\mathrm{XX}$}

2.1. Los comienzos de los estudios de las lenguas fragmentarias tienen una naturaleza eminentemente textual (es decir, que están principalmente centrados en el texto) y, con frecuencia, se reconoce en ellas una intención enfocada básicamente al desciframiento o a la traducción. ${ }^{6}$

La lengua que cuenta con un estudio más longevo es el etrusco, seguida por las sabélicas o las hispánicas y la lengua gala. Prueba de ello son las múltiples traducciones que se hicieron del etrusco a comienzos del siglo XX, fruto del agotamiento del método combinatorio (Benelli 2017, 100), entre las que podemos destacar las propuestas de Vetter (Vetter 1923; 1937), así como algunos de los corpora más precoces: el Corpus Inscriptionum Italicarum (Fabretti 1867), la obra Über die Sprache der Etrusker (Corssen 1874) y, para la península ibérica, los Monumenta Linguae Ibericae (Hübner 1893). A modo de coincidencia y de anécdota, cabe señalar, además, que estas obras comparten la idea de que en la península itálica y en la ibérica se hablaba una sola lengua (respectivamente, panitálica y panhispánica), así como unas primeras propuestas de transcripción / traducción de los textos vernáculos.

Igualmente, el galo, que servía parcialmente para la reconstrucción de una gramática comparada celta (Pedersen 1909-1913), se consideraba como la única lengua antigua dentro de Galia, opinión que duró hasta los descubri-

6 De ahí el interés que suscitaban las inscripciones bilingües, ver Estarán 2016, 16. 
mientos de los materiales aquitanos (Luchaire 1876-1877; Michelena 1954; Gorrochategui 1984) o los narbonenses denominados ligures por Untermann (1969), antes de la llegada de los iberos.

En cualquier caso, como decimos, las obras pioneras sobre los antiguos textos europeos, publicadas entre finales del siglo XVIII y a lo largo del siglo XIX, fueron elaboradas por filólogos cuyo interés era profundizar en sus características lingüísticas. La idea que subyace en todas ellas era que la compilación de epígrafes era el primer paso necesario para obtener material lingüístico, y así hemos de entender volúmenes como el de L. Lanzi, ${ }^{7}$ Saggio di Lingua Etrusca e di altre antiche d'Italia (Lanzi 1789); el de T. Mommsen, Die unteritalischen Dialekte (Mommsen 1850), donde las transcripciones de las inscripciones aparecen en el texto ejemplificando fenómenos lingüísticos y se seleccionan algunos de los epígrafes más llamativos para las tablas de ilustraciones del final.

Pronto surgió la necesidad de compilar todo el material textual existente, en parte debido a la influencia de los grandes corpora epigráficos, el Corpus Inscriptionum Graecarum y el Corpus Inscriptionum Latinarum, cuyo primer volumen salió a la calle en 1853. Este carácter es patente en monumentales obras como el Corpus Inscriptionum Italicarum (Fabretti 1867) y sus apéndices (obra de Gamurrini) y, posteriormente, en el Corpus Inscriptionum Etruscarum, publicado gracias a la iniciativa de Carl Pauli, iniciado a partir de 1885, y en los Monumenta Linguae Ibericae (Hübner 1893). Hay que mencionar paralelamente el interés de la escuela alemana tanto por la propia lengua alemana (normativización, dialectología...) como por otras lenguas, europeas o no: J. C. Adelung había publicado ya en 1806 el primer volumen de su Mithridates (Adelung 1806-1817), en el que recopilaba testimonios sobre las lenguas asiáticas. El resto de volúmenes fueron viendo la luz póstumamente con la ayuda de Vater y de von Humboldt. Los corpora epigráficos antes mencionados supusieron la compilación sistemática y ordenada de todos los materiales textuales de las lenguas en cuestión, un enorme avance que dio lugar a los progresos que tuvieron lugar en la siguiente etapa. De momento, en años inmediatamente posteriores a estas colecciones de inscripciones, vieron la luz capitales obras dedicadas al estudio lingüístico de lenguas fragmentariamente atestiguadas como la de von Planta, Grammatik der oskisch-umbrischen

7 Considerado el padre de la Etruscología científica (Della Fina 2017, 64). Su principal contribución fue el casi definitivo desciframiento del alfabeto etrusco; pero también sentó las bases para conocer la gramática y la fonética de esta lengua. 
Dialekte (Von Planta 1892-1897), donde encontramos un capítulo llamado "Sammlung der Inschriften" de cien páginas en un libro de dos volúmenes con seiscientas y ochocientas páginas respectivamente; o la citada de Corssen que, pese a estructurarse casi por géneros epigráficos, el contenido expone eminentemente datos lingüísticos. ${ }^{8}$ Como mucho, estas pioneras obras contienen lo que hoy consideramos editiones minores de las inscripciones. Podría decirse que una excepción es la obra de Conway (1897), cuyo primer volumen está dedicado exclusivamente a los testimonios epigráficos y numismáticos de los antiguos "dialectos itálicos", y en él se encuentran los datos básicos sobre el soporte y el contexto arqueológico de hallazgo de los epígrafes.

2.2. En 1932 Buonamici publicó Epigrafia Etrusca, una obra que podría considerarse una transición a la siguiente etapa, ya que su autor tuvo la sensatez de huir de los postulados nacionalistas que afectaban a la Etruscología coetánea para aproximarse a las inscripciones de forma positivista (de forma similar a como lo hacemos actualmente) y hacer salir a esta disciplina del "callejón sin salida" en el que estaba situada, empeñada en conseguir traducciones prácticamente inútiles desde el punto de vista científico (Buonamici 1932). ${ }^{9}$ Las palabras de René Cagnat habían causado una gran impresión entre los estudiosos de la época: "lépigraphie n'est pas une science à part, se suffisant à elle-même et sans point de contact avec le faisceau des connaissances qui forment le fond de nos études. Ce riest, à vrai dire, que l'un des éléments, mais c'est un élément essentiel de la philologie, l'une des sources auxquelles doit puiser quiconque veut connaître la religion, les lois, l'histoire politique, la vie privée et le langage des anciens. ${ }^{10}$ La Epigrafía toma carta de naturaleza.

8 Por poner un ejemplo, el capítulo "Weihinschriften, Stiftungs- und Schenkungsurkunden, Künstlerinschriften und verwandte Sprachdenkmäler" se divide en los siguientes apartados: "Inschriften mit Casusformen von Nominalstämmen", "Inschriften mit Verbalformen" e "Inschriften mit Pronominalformen".

9 Benelli 2017, 100 titula "In the dead end" a este apartado de su capítulo de Etruscology.

10 "La Epigrafía no es una ciencia aparte, suficiente por ella misma y sin punto de contacto con el grueso de conocimientos que forman el fondo de nuestros estudios. A decir verdad, no es más que uno de los elementos, pero es un elemento esencial de la Filología, una de las fuentes a las que debe recurrir cualquiera que quiera conocer la religión, las leyes, la historia política, la vida privada y la lengua de los antiguos" (Cagnat 1914, XIII-XIV). 


\section{Colecciones de datos. Desciframientos, descubrimientos y pro- liferación de corpora epigráficos durante la segunda mitad del siglo XX}

3.1. Los imprescindibles trabajos dedicados a la colección sistemática de inscripciones dieron paso inevitable y naturalmente a publicaciones de índole más interpretativa y de puesta en valor del epígrafe en su integridad y no solo como un texto privado de soporte y contexto epigráfico. Esta transformación comenzó a mediados del siglo XX, gracias a las capitales aportaciones de maestros como Susini o Alföldy, o Navascués en España, en el ámbito de la epigrafía latina, que incidieron en la importancia de estudiar de forma conjunta los aspectos materiales y los textuales de los epígrafes. ${ }^{11}$

J. M. de Navascués reivindicó en su discurso de entrada a la Real Academia de la Historia, El concepto de la Epigrafía. Consideraciones sobre la necesidad de su ampliación (Navascués 1953), el papel de la epigrafía como disciplina capital para entender el pasado grecorromano $\mathrm{y}$, además, la necesidad de interpretar las inscripciones en todos sus aspectos: "La inscripción es, pues, como todo escrito, un cuerpo físico en el cual se funden en un solo ser la escritura y su soporte, el cual es a su vez la expresión plástica de un lenguaje y de un pensamiento". ${ }^{12}$ Mediante quizás su obra más célebre, Il lapicida romano, G. Susini supo poner en valor la materialidad de la inscripción (Susini 1966). En ella, evocando el ideario de J. M. de Navascués, recuerda que la inscripción no puede considerarse de forma independiente del monumento sobre la que figura. Por lo que respecta a G. Alföldy, su obra Römischen Inschriften von Tarraco, cuya primera edición fue publicada en 1975, fue y sigue siendo un referente para la edición de conjuntos epigráficos. De hecho, "el trabajo epigráfico de Géza Alföldy constituye un modelo magistral por su rigor en la edición, la atención a soportes y contexto arqueológico, la precisión en la datación y el cuidado por los aspectos prosopográficos, insertando el epígrafe siempre en su contexto histórico y tomando en consideración su papel como medio de comunicación social” (Beltrán 2012).

3.2. Mientras tanto, en el campo de las lenguas de atestación fragmentaria seguían produciéndose sobresalientes trabajos, no solo de perfil eminentemente lingüístico-filológico, como los de E. Vetter y M. Lejeune, sino también

11 Vale la pena señalar que Gamurrini había sido un pionero en este sentido, que ya había avanzado este enfoque en el siglo XIX (Benelli 2017, 97).

12 Sobre este discurso y la figura de Navascués ver De Santiago 2004 y Ramírez 2005. 
otros, como los de M. Pallottino y J. Untermann, que destacaron por subrayar el importante papel del contexto arqueológico, el soporte del epígrafe y los aspectos materiales de manera más marcada que sus contemporáneos colegas estudiosos de la epigrafía latina y griega, probablemente inspirados en el espíritu de los cambios que estaban aconteciendo en la epigrafía latina, pero sobre todo porque los textos en lenguas fragmentarias se comprenden en términos generales mucho peor que los que están escritos en lenguas clásicas, y es necesario un aporte adicional de datos, que se hallan en los elementos extratextuales.

E. Vetter, cuya primera producción, centrada en los significados de las palabras etruscas, ya se ha mencionado en la fase anterior, publicó en 1953 una obra capital para el conocimiento de las lenguas itálicas, Handbuch der italischen Dialekte (Vetter 1953). ${ }^{13}$ La novedad de este volumen reside en la colección de todas las inscripciones conocidas hasta la fecha que estuvieran redactadas en lenguas sabélicas, y muchas de ellas contienen una (escueta) descripción arqueológica. Además, aunque las inscripciones se clasifican por lenguas, dentro de cada lengua, por yacimientos, hay cierta intención de clasificación interna dentro de cada sitio arqueológico por géneros epigráficos o por soportes. ${ }^{14} \mathrm{El}$ "complemento" ${ }^{15}$ de esta obra llevada a cabo por Paolo Poccetti veinticinco años después incorpora algunos significativos avances en la edición de los materiales itálicos consistentes en el cuidado uso de diacríticos (inspirados por la obra Inscriptiones Latinae Liberae Rei Publicae de Degrassi, publicada en 1965) y la descripción más precisa del lugar de hallazgo de la inscripción y del soporte. A nuestro modo de ver, un avance sustancial con respecto de la obra de Vetter que ofrece Paolo Poccetti es la importancia que se le otorga a la autopsia, ya que el mismo autor indica en su prólogo que se realizó allí donde fue posible (Poccetti 1979).

13 Contemporáneo de la publicación de la importantísima colección Fröhner (Lejeune 1953).

14 Por ejemplo, las inscripciones pompeyanas se estructuran en "Bauinschriften", "Aufgemalte Inschriften", "Stempeln auf Ziegeln und Tongefäßen" у "Griffelinschriften auf Außenund Innenwänden"; mientras que las inscripciones faliscas de Falerii están organizadas como "Gefäßinschriften aus der Altstadt" y "Grabschriften aus den Nekropolen und ihrer Umgebung", categoría dentro de la cual hallamos "Weihinschriften aus dem eigentlichen Stadtgebiet", "Grabschriften aus der Nähe der Stadt" e "Inschriften aus Grabkammern der Gegend im Norden von S. Maria di Falleri". 
En la inmensa obra de Michel Lejeune también se refleja esta evolución hacia una edición o comentario de los epígrafes con mayor hincapié en cuestiones no textuales: desde Celtiberica, publicado en 1955 (Lejeune 1955) hasta el Recueil des Inscriptions Gauloises, que se comentará más adelante, la progresión en este sentido queda meridianamente clara, pasando por el Manuel de la langue vénète (Lejeune 1974), obra que, por otra parte, no podría haberse llevado a cabo sin otro mayúsculo libro dedicado a la edición de epígrafes paleoeuropeos: La lingua venética (Pellegrini y Prosdocimi 1967), cuyo título, pese a estar inclinado hacia cuestiones lingüísticas, esconde al lector un fantástico tratamiento de las inscripciones como objetos arqueológicos. Finalmente, como puede percibirse en una de sus obras más tardías, la dedicada al santuario lucano de Rossano di Vaglio, ya contiene una significativa carga de datos arqueológicos y epigráficos (Lejeune 1990).

En la década de los 40 del siglo XX se publicó la obra de Massimo Pallotino, que podría considerarse la "figura central de la Etruscología del siglo XX". ${ }^{16}$ Además de dirigir las excavaciones que dieron lugar al descubrimiento de las láminas bilingües de Pyrgi, así como la monumental obra Thesaurus Linguae Etruscae, demostró en su obra Etruscologia (Pallottino 1942) los beneficios de la confluencia entre arqueología, epigrafía y lingüística. Pese a que no se trata de un corpus epigráfico, su manera de tratar las inscripciones etruscas en Etruscologia tiene en consideración el soporte como un elemento intrínseco del objeto epigráfico. Fue gracias a él (y a sus valiosísimos contemporáneos Colonna, Cristofani, De Simone o Rix) como nació la disciplina moderna de la Epigrafía Etrusca entre los años 1968 y $1970 .{ }^{17}$

3.3. 1942, además, fue un año verdaderamente afortunado para los progresos en la Paleohispanística: Manuel Gómez Moreno ingresó en la Real Academia de la Lengua con su revolucionario discurso "Las lenguas hispánicas" (Gómez Moreno 1942), sentando cátedra y dando forma casi definitiva a su propuesta de desciframiento del signario paleohispánico. Gracias a este sustancial avance pudieron comenzar a comprenderse los antiguos textos de la península ibérica, una línea de investigación que fue una de las elegidas por un joven Jürgen Untermann, que ya en los años 60 estaba dedicado a las lenguas fragmentarias de la península itálica (Untermann 1961a; De Simone y Untermann 1964), y que estaba llamado a ser uno de los padres de los estudios paleohispanísticos. Su obra Monumenta Linguarum Hispanicarum (1975, 
volumen $1 ; 1980$, volumen $2 ; 1990$, volumen $3 ; 1997$, volumen 4) dio un giro a las publicaciones epigráficas precedentes dedicadas a la epigrafía paleohispánica. Solo por mencionar algunos de los aportes metodológicos de los $M L H,{ }^{18}$ en primer lugar, Untermann consideró las inscripciones sobre moneda como parte del corpus de textos de las antiguas lenguas de Hispania, cuando siempre habían quedado relegadas únicamente a los estudios numismáticos. ${ }^{19}$ Segundo, incluidas en una estructura geográfico-lingüístico-cultural, agrupó por fichas los textos paleohispánicos conocidos hasta la fecha. En estas schedae Jürgen Untermann consiguió un mayor equilibrio entre la información arqueológica, lingüística e histórica de la pieza (equilibrio que no se consigue, por ejemplo, en los volúmenes antiguos del $C I L$ ), razón por la cual se han convertido en una inspiración para corpora epigráficos publicados con posterioridad $(B D H$; Simón 2013; Estarán 2016; Jordán 2019), especialmente en lo que respecta a la introducción de fotos, ilustraciones o calcos de las piezas. La de Untermann es la primera compilación epigráfica elaborada con este enfoque dedicada a las lenguas fragmentarias.

3.4. Esta nueva forma de ver las inscripciones como fuentes históricas integrales, por así decirlo, como objetos arqueológicos que contienen un texto, generó una nueva línea de aproximación a la cuestión de corte más histórico-cultural: la del estudio de la cultura escrita y, concretamente, de la epigrafía como medio de comunicación. Géza Alföldy, de nuevo, centrándose especialmente en el poder de las inscripciones como instrumentos de autorrepresentación y de propaganda del régimen de Augusto (Alföldy 1991) fue el impulsor de esta perspectiva tan fecunda que poco después dio lugar a una larga lista bibliográfica de la que aquí mencionaremos tan solo una monografía precisamente dedicada a Roma y el nacimiento de la cultura epigráfica en Occidente (Beltrán 1995), en la que, naturalmente, participó G. Alföldy con un capítulo dedicado a la costa levantina (Alföldy 1995), y que contiene otros trabajos que aún hoy siguen vigentes.

Este volumen, editado por F. Beltrán, podría entenderse como una obra pionera de los estudios sobre cultura escrita en la Antigüedad, en tanto que contribuyó significativamente a aportar un enfoque sociológico a la epigrafía como medio de comunicación social. Además, y en particular para lo que nos

18 Este no es el lugar para explicar las innumerables aportaciones teóricas de los $M L H$ de Untermann a nuestro conocimiento de las lenguas paleohispánicas.

19 Modelo que posteriormente siguieron B. Fischer y J. B. Colbert de Beaulieu en el cuarto volumen del $R I G$, publicado más de veinte años después. 
interesa aquí, es muy relevante el hecho de que fue la primera obra en la que se pusieron al mismo nivel varias culturas epigráficas paleoeuropeas (especialmente las paleohispánicas) y la latina, puesto que el libro fue concebido con el espíritu de abordar todas las inscripciones contemporáneas como un fenómeno único, independientemente de la lengua en que estuvieran escritas. En este sentido (y no en la selección de conjuntos epigráficos), este volumen es claramente precursor del concepto de AELAW.

3.5. Volviendo al final del punto 3.3, puede afirmarse que, gracias a estos esfuerzos por considerar la inscripción como un "objeto mixto", por así decirlo, compuesto por elementos históricos y lingüísticos, se han podido lograr, a su vez, considerables progresos en la reconstrucción histórica de los pueblos paleoeuropeos y sus aspectos sociales $y$, naturalmente, en nuestro conocimiento de sus lenguas y sistemas de escritura.

Desde el cambio en la valoración de los aspectos materiales de las inscripciones prácticamente no ha habido marchas hacia atrás y este tipo de aproximación se ha seguido mayoritariamente en la actualidad. Sin embargo, no es la única utilizada; podemos fijarnos como ejemplo en las obras de H. Rix, Etruskische Texte y Sabellische Texte (Rix 1991; Rix 2002 respectivamente $^{20}$ ), que están concebidas como editiones minores de los textos etruscos y sabélicos, estructurados de forma un tanto confusa en géneros textuales dentro de su obra. $^{21}$

3.6. También podemos observar algo similar en el ámbito de la lengua gala; después de trabajos pioneros como el de Dottin, ha tenido lugar una importante ampliación de documentación epigráfica, y gracias a la atención que le dedicaron estudiosos como L. Weisherber (a quien debemos la acuñación del término Festlandkeltisch o 'celta continental'), o J. Whatmough, fue tomando el galo un papel cada vez más importante en el seno de los dialectos celtas (Dottin 1920; Weisherber 1931; Whatmough 1970). Con la reciente aparición de textos de longitud respetable, como las inscripciones de Chamalières o Larzac en el dominio galo, y la identificación del lepóntico como dialecto independiente del celta continental, gracias a Lejeune 1971, los documentos

20 Versión actualizada de Etruskische Texte en Meiser 2014.

21 Helmut Rix combinó la aproximación lingüística a los textos y las editiones minores con interesantísimos trabajos sobre onomástica, como su capital Das etruskische Cognomen: Untersuchungen zu System, Morphologie und Verwendung der Personennamen auf den jüngeren Inschriften Nordetruriens (Rix 1965) o "Zum Ursprung des römisch-mittelitalischen Gentilnamensystems" (Rix 1972). 
celtas más antiguos han sido elevados últimamente hasta el s. VI a.C. El RIG, iniciado por Lejeune y continuado por Lambert, consta de cuatro volúmenes (el segundo subdividido en dos fascículos); el primero de ellos fue publicado en 1985 (volumen 2, en 1988 y 2002; volumen 3, en 1986 y volumen 4, en 1998), y en todos ellos se explican los aspectos extratextuales de las inscripciones de forma muy amplia, bien es verdad que con un corte mucho más filológico que el de $M L H$, cosa que queda patente en el tipo de ficha.

3.7. Aparte del citado M. Lejeune, en cuya obra epigráfica estudió la reforma alfabética del alfabeto osco nacional, el uso del alfabeto griego para algunas inscripciones oscas, el sistema del alfabeto véneto o la adopción del etrusco septentrional para el lepóntico, las lenguas itálicas también se han beneficiado de un gran avance más reciente al ser descifrada la escritura utilizada para las inscripciones sudpicenas (Marinetti 1985), datadas entre el siglo VI y el año 300 a.C. y con isoglosas comunes con el umbro. Este avance se ha producido a la vez que el descubrimiento de nuevos textos epigráficos, ${ }^{22} \mathrm{mu}$ chas veces con un trabajo previo de desciframiento de sistemas de escritura, labor muy importante incluso en Italia, a pesar de que en esta región todas las escrituras provienen en última instancia de modelos griegos.

3.8. La publicación de los sucesivos volúmenes de los Monumenta Linguarum Hispanicarum resultó un acicate para los estudios paleohispanísticos, puesto que todo el material estaba reunido en una sola obra (desde la publicación de los $M L H$ las inscripciones paleohispánicas se compilan en De Hoz y Luján 2001 y en el resto de Chronicae Epigraphicae de la revista Palaeohispanica). Debido a la particular naturaleza de las dos principales lenguas paleohispánicas, el ibérico y el celtibérico, la producción relativa a cada una de ellas se ha adaptado a sus respectivas necesidades. Mientras que es posible, para el celtibérico, una vez recopilado el material, una aproximación de tipo lingüístico, no lo es en cambio para el ibérico, puesto que todavía se desconocen muchas de sus características gramaticales. Por tanto, el ibérico se aborda mediante onerosas obras de tipo léxico (Siles 1985; Velaza 1991; MLH V.2). La lengua y la epigrafía celtibéricas, en contraste, han sido objeto de varias obras en las que se ha podido ahondar sobre cuestiones lingüísticas y gramaticales, si bien D. Wodtko también acometió la ingente tarea de publicar un diccio-

22 Hay que reconocer el trabajo de algunas revistas donde se han focalizado estudios epigráficos (como Studi Etruschi), tanto para dar a conocer inscripciones etruscas como novedades de toda la Italia prerromana. Otra revista interesante es Revue des Études Latines, donde el mismo Lejeune publicó innumerables artículos sobre alfabetos itálicos. 
nario de términos celtibéricos ( $M L H$ V.1). Tras Francisco Villar o Javier de $\mathrm{Hoz},{ }^{23}$ ha sido Carlos Jordán uno de los autores que más trabajo ha dedicado al conocimiento de esta lengua: además de numerosos artículos y obras de síntesis, ${ }^{24}$ tres volúmenes (Jordán 1998; 2004; 2019) en los que se aprecia una clara evolución hacia el aprovechamiento integral del epígrafe o la leyenda monetal como fuente del conocimiento de la lengua y la cultura celtibéricas. Mientras que los dos primeros están eminentemente centrados en la lengua celtibérica (y su título así lo anuncia), el tercero amplía su ámbito de interés a la Lengua y epigrafía celtibéricas, y la prueba es que más de setecientas páginas de las mil cincuenta de esta obra están dedicadas a los documentos en lengua celtibérica.

\section{Interacción con los datos. El siglo XXI: las lenguas paleoeuropeas en la era digital.}

4.1. La concepción de la inscripción como un objeto analizable desde perspectivas interdisciplinares, con algunos antecedentes claros ya comentados, ha permitido avanzar mucho en el estudio de estas lenguas antiguas al enriquecer los estudios más nuclearmente etimológicos con otros aspectos más contextuales. Es básico conocer el contexto y la finalidad de una inscripción para interpretar mejor el significado de su contenido. En ocasiones es el soporte escriturario el que ayuda a fijar la función del texto, con lo que los paralelos de soporte que observamos en todo el mundo antiguo resultan de gran ayuda en este análisis. También la alternancia entre distintos tipos de escritura, entre distintas lenguas o variantes de lenguas y, en definitiva, las cuestiones que tienen que ver con la sociolingüística serán de obligada aplicación para un texto antiguo.

Desde el principio de este tipo de estudios, la onomástica ha desempeñado un papel crucial en el desciframiento de sistemas de escritura (caso del cuneiforme persa, el jeroglífico egipcio o la escritura ibérica, las lenguas del norte de Italia, ${ }^{25}$ el britónico o el goidélico; ver nota 21); por ello debe ocupar también un espacio predominante entre las lenguas paleoeuropeas.

23 Por ejemplo, Villar 1995a; 1995b; 1997; 2007 o De Hoz 1986; 1988; 1995.

24 Sin contar su publicación en e-keltoi (Jordán 2007), el Booklet de la serie $A E L A W$ de la que es coautor (Beltrán y Jordán 2016) y un pequeño manual en prensa (Jordán e. p.).

25 J. Untermann estableció la presencia de diferentes lenguas (consecuentemente vinculadas con diversas culturas epigráficas) en el norte de Italia basándose en áreas antroponomásticas (Untermann 1959; 1960; 1961b). 
Valdría citar la relevancia que ha tenido en la península ibérica la asignación del elemento onomástico - briga a las lenguas célticas (a causa de su exclusiva evolución fonética), estableciendo así una clara distinción frente al mundo no indoeuropeo peninsular. Por otro lado, la presencia de elementos onomásticos en lenguas sin descifrar (como el ibérico) supone una ayuda para navegar con alguna garantía por textos que, de otra manera, resultarían absolutamente opacos.

4.2. Podría afirmarse que la mayoría de ediciones epigráficas digitales (al menos, de las dedicadas a las Restsprachen) son herederas de Untermann, especialmente el Banco de Datos Hesperia, que ha dado cobertura a todas las lenguas peninsulares, para las que se ha convertido en una referencia. El proyecto Hesperia, ideado e iniciado por Javier de Hoz en los años 90 del siglo $\mathrm{XX}$, abarca todas las lenguas hispánicas de la antigüedad a través de la recogida, almacenamiento y gestión de sus testimonios, tanto directos como indirectos. Los materiales directos (inscripciones en lengua indígena, mixta o bilingües) son el objetivo fundamental del proyecto y constituyen, además, una actualización de los $M L H$ de Untermann: las inscripciones directamente elaboradas por los hablantes indígenas (cualquier tipo de inscripción pública o privada en cualquier soporte) se organizan de una manera geográfica atendiendo a límites administrativos modernos. Cada ficha incluye la descripción de la inscripción desde un punto de vista interno (características físicas del soporte, medidas, texto, lengua y cronología) y externo (condiciones del hallazgo, localización actual...), con lo que se proporcionan los datos suficientes para la consulta comparada de todo el material, que sirvan, al final, para la elaboración de mapas o cuadros comparativos. La numismática tiene un modelo individual de gestión, puesto que se agrupa por las emisiones antiguas, pero da lugar igualmente a las mismas posibilidades de consulta y gestión de la información. Los materiales indirectos (onomástica, glosas...) están también presentes en el proyecto.

La elaboración del modelo definitivo de Base de Datos no ha sido fácil: ha sido necesario tomar decisiones sobre cuestiones de fondo en el funcionamiento de la base (la cimentación del edificio): una de ellas es definir el número total de lenguas y sistemas de escritura ( $\mathrm{y}$ todas las variantes de ambas) con las que tenemos que trabajar. Establecer un consenso sobre este primer paso asegura los siguientes, como pueden ser el sistema de transcripción del texto de acuerdo con la lengua de la que se trate y el sistema de escritura utilizado. Cada ficha toma en consideración un texto y no su soporte (aunque se aporta 
toda la información oportuna sobre él y su contexto de hallazgo), dado que algunas inscripciones se extienden por varias zonas de una misma inscripción y, por otro lado, algunos soportes (parietales, rupestres...) pueden contener más de una inscripción.

Para apreciar esta moderna ampliación de enfoques podemos citar el caso del lusitano, una de las lenguas paleoeuropeas más occidentales, con unos escasos materiales, que ha pasado de ser valorada exclusivamente con ópticas lingüísticas a partir de sus documentos escritos a ser considerada como un modelo de aproximación multidisciplinar desde distintos puntos de vista: el primero es el religioso, por el carácter de todas sus inscripciones. Estas, también, presentan mayoritariamente un aspecto bilingüe en mayor o menor grado, con lo que la sociolingüística puede intervenir para extender la etiqueta de lusitano a inscripciones con carácter bilingüe menos evidente, pero con restos morfológicos incompatibles con el latín (cf. Vallejo 2013). La onomástica, como ya hemos avanzado, resulta una herramienta importante en el estudio de las lenguas, especialmente en esta, a la hora de observar la coincidencia entre el área de dispersión geográfica de los nombres mejor atestiguados y la zona de ampliación propuesta para la extensión de los documentos lingüísticos directos.

En cualquier caso, la solución lexicográfica planteada por el Banco de Datos Hesperia es solo una de las posibles. ${ }^{26}$ Además de Hesperia y de Ogham in $3 D^{27}$ (que cronológicamente queda fuera de nuestro ámbito de interés), solo otras dos bases de datos de lenguas paleoeuropeas han sido realizadas hasta la fecha, LexLep y $T I R ; ;^{28}$ aunque está previsto que en los próximos años se publique $M I B$, una base de datos numismática realizada por P. P. Ripollès y $\mathrm{M}$. Gozalbes, que permitirá avanzar sustancialmente en el estudio de las leyendas monetales de la península ibérica hasta el año 44 a. C., y RIIG, un proyecto coordinado por C. Ruiz Darasse, gracias al cual se editarán digitalmente las inscripciones en lengua gala.

LexLep nació en 2009, coordinado por David Stifter de la Universidad de Maynooth (pero con base en Viena) con el espíritu de ser un diccionario

26 Sobre la lexicografía computacional de Hesperia, ver Estarán et al. 2019, 43-44. Ver De Santis y Rossi 2019 para una puesta en común completa de múltiples bases de datos epigráficas digitales, los problemas particulares a los que se han de enfrentar y sus soluciones lexicográficas.

28 Sobre el estado de la cuestión de la edición digital de lenguas paleoeuropeas, $c f$. Estarán e. p. 
etimológico interactivo y en línea de la lengua lepóntica y del galo cisalpino, y ha acabado convirtiéndose en una utilísima base de datos con una completa información sobre los aspectos materiales y contextuales de las inscripciones. El TIR, coordinado por Stephan Schumacher, Sindy Kluge y Corinna Salomon, y también con base en Viena, se abrió al público en 2013. Estaba concebido de la misma manera que LexLep, es decir, basado en MediaWiki. En verano de 2015, prácticamente todas las inscripciones réticas estaban dentro del sistema (previa autopsia) y, al igual que en el caso del Léxico Lepóntico, la información estaba estructurada en páginas dedicadas a palabras, morfemas, fonemas y caracteres, así como en resúmenes de temas relevantes relacionados con la epigrafía rética. Tanto TIR como LexLep aspiraban a crear una colección y edición completa de todos los textos escritos en los llamados alfabetos itálicos septentrionales y ambos proyectos tenían la intención de crear nuevos estándares al aplicar herramientas relativamente punteras a los estudios lingüísticos, y además era uno de sus objetivos implantar MediaWiki para este tipo de bases de datos. Sin embargo, este soporte se ha visto superado por la tecnología de Linked Open Data que está utilizándose en otras bases de datos epigráficas. $^{29}$

Todas estas disciplinas, junto con otras como las más puramente epigráficas, históricas o arqueológicas han potenciado enormemente el estudio de algunas áreas. La correcta combinación de estos estudios con las posibilidades tecnológicas a nuestro alcance favorece el desarrollo de un tipo de estudios cuyas posibilidades no podíamos prever hace tan solo unos pocos años.

Resultado de estos avances tecnológicos son obras de gran calibre como los volúmenes de Historia lingüística de la Península Ibérica en la Antigüedad (De Hoz 2010; 2011), la última obra sobre Lengua y epigrafía Celtibéricas (Jordán 2019), o la finalización del volumen denominado por Untermann Die vorrömische einheimische Toponymie des antiken Hispanien (MLH VI) por citar algunos ejemplos de la península ibérica.

Fuera de ella, en regiones que aún carecen de una base de datos epigráfica elaborada con criterios científicos, siguen editándose monumentales diccionarios y corpora elaborados gracias a la cuidadosa y paciente compilación de datos por parte de sus editores, como el diccionario de osco-umbro de Untermann (2000) o las Imagines Italicae (Crawford 2011), que han supuesto la

$<$ https://wiki.digitalclassicist.org/Category:EpiDoc $>$. 
primera colección de epigrafía osco-umbra desde los complementos de Vetter elaborados por Poccetti (1979), obra en la que por primera vez se incluyen imágenes de las piezas; Monumenta Linguae Messapicae (De Simone y Marchesini 2002); Monumenta Linguae Raeticae (Marchesini y Roncador 2015).

\section{Criterios comunes para el futuro. Hacia una unificación del estudio de las lenguas paleoeuropeas: la aportación de AELAW}

5.1. La base de datos de AELAW es, en consecuencia, hija de su tiempo y de sus circunstancias. Nacida como parte de la red COST Ancient European Languages and Writings, dirigida por F. Beltrán, tenía como objetivo primordial censar las antiguas lenguas europeas y, adicionalmente, sus textos, razón por la cual esta base de datos, consultable en el sitio web <aelaw.unizar.es $>$, diferencia languages (<aelaw.unizar.es/database/languages $>$ ) de inscriptions (< $<$ aelaw.unizar.es/database/inscriptions $>$ ). ${ }^{30}$

Si tenemos en cuenta que, de los c. 17.000 textos censados, $c .14 .000$ proceden de la península itálica y $c$. 2500, de la ibérica y $c$. 800, de Francia, es más fácil comprender por qué se han podido individualizar 21 lenguas en la primera, 5 en la segunda y 1 en la tercera. La cantidad de documentación conservada en cada caso condiciona totalmente las aproximaciones de la investigación actual: por poner un ejemplo, mientras que en Italia es posible hacer un corpus de las inscripciones en lengua mesápica, otro de las sabélicas, otro de las etruscas, etc., que aunque son contemporáneas, no se interpretan como tales sino solo en estudios separados, en la antigua Galia, que es un territorio más vasto, nos vemos obligados a recoger e interpretar las inscripciones galas como si hubiera un continuum lingüístico desde Marsella hasta Bélgica, cuando seguramente habría dialectos galos o bolsas lingüísticas diferentes. Gracias a los censos de AELAW se han puesto de relieve estas abismales desigualdades en las fuentes epigráficas, que, desde luego, también ocurren en la península ibérica y en las regiones de la "Europa silenciosa". ${ }^{11}$ Se adopta una misma me-

30 Estos censos han sido confeccionados por varios miembros de la red a lo largo de sus Short Term Scientific Missions (Carlos Bueno, Javier Herrera, Gabriela de Tord, Federica Fumante, Ángel López Chala, Aránzazu López Fernández, Noemí Moncunill, Lorenzo Muzzi, Corinna Salomon, Víctor Sabaté, Ignacio Simón) y supervisados por los responsables de cada equipo.

31 Se tienen también en consideración lenguas documentadas de forma indirecta en inscripciones latinas, como el tracio, el vascónico-aquitano, o el ligur. Un caso especial es el lusitano, ya que su registro combina inscripciones íntegramente escritas en ella con inscripciones latinas que contienen interferencias morfológicas de esta lengua. 
todología que es independiente de la lengua de los textos: se pretende, como ya hizo el pionero J. Untermann en $M L H$, un resultado interlingüístico, centrado en las experiencias epigráficas y culturales coetáneas.

5.2. La puesta en común de estos datos en foros nutridos de investigadores dedicados a los diversos conjuntos de antiguas inscripciones de Europa ha llevado a la creación de un término que permitiera referirse a todas ellas en su conjunto y que no tuviera connotaciones de dependencia de la cultura romana (como puede tenerlas el término "prerromanas"), de modo que, siguiendo la iniciativa de F. Beltrán, se habló de "lenguas y escrituras paleoeuropeas", un término que, inevitablemente, es heredero de "lenguas y culturas paleohispánicas".

5.3. Solo después de un recorrido historiográfico como el descrito en las páginas anteriores se comprende el enfoque de $A E L A W$ : la inscripción entendida en su conjunto texto-soporte-contexto como objeto principal de estudio. Esto ha dado lugar a un acercamiento de estudiosos de muy diversa formación (histórica, filológica) para trabajar de forma conjunta. Como resultado de los avances descritos a lo largo de la presente contribución, los textos célticos, que tradicionalmente se estudian desde un punto de vista más filológico son analizados también desde el punto de vista epigráfico y también al contrario: otras culturas epigráficas cuya lengua se desconoce más, y que tradicionalmente se estudiaban desde un enfoque eminentemente arqueológico, como el ibérico o el rético, están comenzando a ser estudiadas desde un punto de vista lingüístico, y $A E L A W$ ha contribuido significativamente a asentar estas tendencias. Las palabras que Buonamici publicó, refiriéndose a una lengua indescifrada como el etrusco, hace casi cien años siguen estando, pues, plenamente en vigor: "Ĺepigrafia può fare a meno dell'ermeneutica, ma, per l'Etrusco, l'ermeneutica non può fare a meno dell'epigrafia (...). La ricerca epigrafica (...) è il primo gradino, l'ABC di ogni tentativo ermeneutico che seriamente si voglia intraprendere intorno all'Etrusco" ${ }^{32}$

\section{Abreviaturas}

AELAW: Ancient European Languages and Writings (<aelaw.unizar.es $>$ ).

BDH: Banco de Datos de Lenguas Paleohispánicas Hesperia (<hesperia.ucm.es $>)$.

LexLep: Lexicon Leponticum (<www.univie.ac.at/lexlep/wiki $>$ ).

RIIG: Recueil Informatisé des Inscriptions Gauloises (<riig.huma-num.fr $>$ )

TIR: Thesaurus Inscriptionum Raeticarum (<www.univie.ac.at/raetica/wiki $>$ )

32 Extraído de Benelli 2017, 100, n. 10. 


\section{$\begin{array}{llllllllllll}\mathbf{B} & \mathbf{I} & \mathbf{B} & \mathbf{L} & \mathbf{I} & \mathbf{O} & \mathbf{G} & \mathbf{R} & \mathbf{A} & \mathbf{F} & \mathbf{I} & \mathbf{A}\end{array}$}

Adelung 1806-1817: J. C. Adelung, Mithridates oder allgemeine Sprachenkunde mit dem Vater Unser als Sprachprobe in beynahe fünfhundert Sprachen und Mundarten, 4 vols. (los volúmenes 2 al 4 se publicaron sobre los manuscritos de Adelung con las adiciones de J. S. Vater y W. von Humboldt), Berlín 1806-1817.

Alföldy 1975: G. Alföldy, Die römischen Inschriften von Tarraco, Berlín 1975.

Alföldy 1991: G. Alföldy, "Augustus und die Inschriften: Tradition und Innovation. Die Geburt der imperialen Epigraphik", Gymnasium 98, 1991, 289-324.

Alföldy 1995: G. Alföldy, "Die Entstehung der epigraphischen Kultur der Römer an der levanteküste”, en: F. Beltrán (ed.), Roma y el nacimiento de la cultura epigráfica en Occidente, Zaragoza 1995, 121-137.

Beltrán 1995: F. Beltrán (ed.), Roma y el nacimiento de la cultura epigráfica en Occidente, Zaragoza 1995.

Beltrán 2012: F. Beltrán, “Géza Alföldy (Budapest 1935 - Atenas 2011)”, Espacio, tiempo y forma. Serie II, Historia antigua 25, 2012, 15-18.

Beltrán y Jordán 2016: F. Beltrán y C. Jordán, Celtibérico: lengua, escritura epigrafía (AELAW Booklets), Zaragoza 2016.

Benelli 2017: E. Benelli, "Approaches to the study of the language", en: A. Naso (ed.), Etruscology, Boston-Berlín 2017, 95-108.

Buonamici 1932: G. Buonamici, Epigrafia Etrusca, Florencia 1932.

Campanile 1983: E. Campanile, "Le Restsprachen e la ricerca indoeuropeistica", en: E. Vineis (ed.), Le lingue indoeuropee di frammentaria atestazione / Die indogermanischen Restsprachen (Atti del Convegno della Società Italiana di Glottologia e della Indogermanische Geschellschaft, Udine, 22-24 sett. 1981), Pisa 1983, 18-28.

Cagnat 1914: R. Cagnat, Cours d’épigraphie latine, París 1914 [4. ${ }^{a}$ ed.].

Conway 1897: R. S. Conway, The Italic Dialects, Cambridge 1897.

Corssen 1874: W. P. Corssen, Über die Sprache der Etrusker, Leipzig 1874.

Crawford 2011: M. H. Crawford, Imagines Italicae: A Corpus of Italic Inscriptions, Londres 2011.

De Hoz 1986: J. de Hoz, "La epigrafía celtibérica”, en: G. Fatás (ed.), Reunión sobre epigrafía hispánica de época romano-republicana (Zaragoza 1983), Zaragoza 1986, 43-102.

De Hoz 1988: J. de Hoz, "La lengua y la escritura de los celtíberos", en: F. Burillo, J. A. Pérez y M. L. Sus (eds.), Celtíberos, Zaragoza 1988, 145-153.

De Hoz 1995: J. de Hoz, "Las sociedades celtibérica y lusitana y la escritura”, AEspA 68, 1995, 3-30.

De Hoz 2010: J. de Hoz, Historia lingüística de la Península Ibérica en la Antigüedad. I. Preliminares y mundo meridional prerromano, Madrid 2010.

De Hoz 2011: J. de Hoz, Historia lingüística de la Península Ibérica en la Antigüedad. II: El mundo ibérico prerromano y la indoeuropeización, Madrid 2011.

De Hoz y Luján 2001: J. de Hoz y E. Luján, “Inscripciones ibéricas no recogidas en MLH I”, PalHisp 1, 2001, 355-367. 
De Santiago 2004: J. de Santiago, "La epigrafía: evolución conceptual y metodológica", Documenta \& Instrumenta 1, 2004, 203-220.

De Santis y Rossi 2019: A. M. de Santis e I. Rossi (eds.), Crossing Experiences in Digital Epigraphy. From Practice to Discipline, Varsovia-Berlín 2019.

De Simone y Untermann 1964: C. de Simone y J. Untermann, Die messapischen Inscriften. Die messapischen Personennamen, Wiesbaden 1964.

De Simone y Marchesini 2002: C. de Simone y S. Marchesini, Monumenta Linguae Messapicae, Wiesbaden 2002.

Della Fina 2017: G. M. della Fina, “History of Etruscology”, en: A. Naso (ed.), Etruscology, Boston-Berlín, 2017, 95-108.

Dottin 1920: G. Dottin, La langue gauloise, París 1920.

Estarán 2016: M. J. Estarán, Epigrafía Bilingüe del Occidente Romano. El latín y las lenguas locales en las inscripciones bilingües y mixtas, Zaragoza 2016.

Estarán et al. 2019: M. J. Estarán, F. Beltrán, E. Orduña y J. Gorrochategui, “Hesperia, a Database for Palaeohispanic Languages; and AELAW, a Database for the Ancient European Languages and Writings. Challenges, Solutions, Prospects", en: A. M. de Santis e I. Rossi (eds.), Crossing Experiences in Digital Epigraphy. From Practice to Discipline, VarsoviaBerlín 2019, 36-48.

Estarán e. p.: M. J. Estarán, "Digital edition of Palaeo-European texts. State of the art", en: I. Velázquez y D. Espinosa (eds.), Epigraphy in the Digital Age: Chances and Challenges of the Recording, Analysis and Dissemination of Epigraphic Texts, Oxford en prensa.

Fabretti 1867: A. Fabretti, Corpus Inscriptionum Italicarum, Florencia 1867.

Gómez Moreno 1942: M. Gómez Moreno, "Las lenguas hispánicas”. Discurso leído ante la Real Academia Española de la Lengua, Madrid 1942.

Gorrochategui 1984: J. Gorrochategui, Onomástica indígena de Aquitania, Bilbao 1984.

Hübner 1893: E. Hübner, Monumenta Linguae Ibericae, Berlín 1893.

ILLRP: A. Degrassi, Inscriptiones Latinae Liberae Rei Publicae, Florencia 1957-1963.

Jordán 1998: C. Jordán, Introducción al celtibérico, Zaragoza 1998.

Jordán 2004: C. Jordán, Celtibérico, Zaragoza 1994.

Jordán 2007: C. Jordán, “Celtiberian”, e-Keltoi: Journal of Interdisciplinary Celtic Studies, 6, 2007, article 17 (<https://dc.uwm.edu/ekeltoi/vol6/iss1/17>).

Jordán 2019: C. Jordán, Lengua y epigrafía celtibéricas, Zaragoza 2019.

Jordán e. p.: C. Jordán, Introduction à leétude de la langue et de l’épigraphie celtibères, Lovaina e. p.

Lanzi 1789: L. Lanzi, Saggio di lingua etrusca e di altre antiche d'Italia per servire alla storia de popoli, delle lingue e delle belle arti, Roma 1789.

Lejeune 1953: M. Lejeune, Collection Froehner: inscriptions italiques, París 1953.

Lejeune 1955: M. Lejeune, Celtiberica, Salamanca 1955.

Lejeune 1971: M. Lejeune, Lepontica, París 1971.

Lejeune 1974: M. Lejeune, Manuel de la langue vénète, Heidelberg 1974.

Lejeune 1990: M. Lejeune, Méfitis d’après les dédicaces lucaniennes de Rossano Di Vaglio, Louvain-la-Neuve 1990.

Luchaire 1876-1877: A. Luchaire, “Les origines linguistiques de l'Aquitaine”, Bulletin de la Société des Sciences, Lettres et Arts de Pau 10, 1876-1877, 349-423 [publicado por separado en Pau 1877]. 
Marchesini y Roncador 2015: S. Marchesini y R. Roncador, Monumenta Linguae Raeticae, Roma 2015.

Marinetti 1985: A. Marinetti, Le iscrizioni sudpicene. I: Testi, Florencia 1985.

Meiser 2014: G. Meiser, Etruskische Texte: editio minor, Zahna-Elster 2014.

Michelena 1954: L. Michelena, “De onomástica aquitana”, Pirineos 10, 1954, 409-455.

MLH I: J. Untermann, Monumenta Linguarum Hispanicarum I. Die Münzlegenden. 1. Text. 2. Tafeln, Wiesbaden 1975.

MLH II: J. Untermann, Monumenta Linguarum Hispanicarum II. Die Inschriften in iberischer Schrift aus Südfrankreich, Wiesbaden 1980.

MLH III: J. Untermann, Monumenta Linguarum Hispanicarum III. Die iberischen Inschriften aus Spanien. 1. Literaturverzeichnis, Einleitung, Indices. 2. Die Inschriften, Wiesbaden 1990.

MLH IV: J. Untermann, Monumenta Linguarum Hispanicarum IV. Die tartessischen, keltiberischen und lusitanischen Inschriften, Wiesbaden 1997.

MLH V.1: D. Wodtko, Monumenta Linguarum Hispanicarum V.1. Wörterbuch der keltiberischen Inschriften, Wiesbaden 2000.

MLH V.2: N. Moncunill y J. Velaza, Monumenta Linguarum Hispanicarum V.2. Lexicon der iberischen Inschriften / Léxico de las inscripciones ibéricas, Wiesbaden 2019.

MLH VI: J. Untermann (†), Monumenta Linguarum Hispanicarum VI. Die vorrömische einheimische Toponymie des antiken Hispanien, Wiesbaden 2018.

Mommsen 1850: T. Mommsen, Die unteritalische Dialekte, Leipzig 1850.

Navascués 1953: J. M. de Navascués, "El concepto de la Epigrafía. Consideraciones sobre la necesidad de su ampliación”. Discurso leído ante la Real Academia de la Historia, Madrid 1953.

Pallottino 1942: M. Pallottino, Etruscologia, Milán 1942.

Pedersen 1909-1913: H. Pedersen, Vergleichende Grammatik der keltischen Sprachen [2 vols.], 1909-1913 [reproducción facsímil, Göttingen 1976].

Pellegrini y Prosdocimi 1967: G. B. Pellegrini y A. Prosdocimi, La lingua venetica, Padua 1967.

Poccetti 1979: P. Poccetti, Nuovi documenti italici a complemento del manuale di E. Vetter, Pisa 1979.

Ramírez 2005: M. Ramírez, "El concepto de Epigrafía. Consideraciones sobre la necesidad de su ampliación, cincuenta años después", Signo. Revista de Historia de La Cultura Escrita 15, 2005, 47-76.

RIG I: M. Lejeune, Recueil des Inscriptions Gauloises, Vol. I. Textes gallo-grecs, París 1985.

RIG II-1: M. Lejeune, Recueil des Inscriptions Gauloises, Vol. II-1. Textes gallo-étrusques, Textes gallo-latins sur pierre, París 1988.

RIG II-2: P.-Y. Lambert, Recueil des Inscriptions Gauloises, Vol. II-2. Textes gallo-latins sur instrumentum, París 2002.

RIG III: P. M. Duval y G. J. Pinault, Recueil des Inscriptions Gauloises, Vol. III. Les calendriers (Coligny, Villards d'Hería), París 1986.

RIG IV: J. B. Colbert de Beaulieu y B. Fischer, Recueil des Inscriptions Gauloises, Vol. IV. Les légendes monétaires, París 1998.

Rix 1965: H. Rix, Das etruskische Cognomen: Untersuchungen zu System, Morphologie und Verwendung der Personennamen auf den jüngeren Inschriften Nordetruriens, Wiesbaden, 1965.

Rix 1972: H. Rix, "Zum Ursprung des römisch-mittelitalischen Gentilnamensystems", ANRW I.2, Berlín 1972, 700-758. 
María José Estarán Tolosa | José María Vallejo

Rix 1991: H. Rix, Etruskische Texte: editio minor, Tübingen 1991.

Rix 2002: H. Rix, Sabellische Texte. Die Texte des Oskischen, Umbrischen und Südpikenischen, Heidelberg 2002.

Siles 1985: J. Siles, Léxico de inscripciones ibéricas, Madrid 1985.

Simón 2013: I. Simón, Los soportes de la epigrafía paleohispánica, Zaragoza-Sevilla 2013.

Susini 1966: G. C. Susini, Il lapicida romano. Introduzione all' epigrafia latina, Bolonia 1966.

Untermann 1959: J. Untermann, "Namenlandschaften im alten Oberitalien (I)”, BzN 10, 1959, 75-159.

Untermann 1960: J. Untermann, “Namenlandschaften im alten Oberitalien (II)”, $B z N$ 11, 1960, 273-318.

Untermann 1961a: J. Untermann, Die venetischen Personennamen, Wiesbaden 1961.

Untermann 1961b: J. Untermann, "Namenlandschaften im alten Oberitalien (III)", BzN 12, 1961, 1-30.

Untermann 1969: J. Untermann, 1969, “Lengua gala y lengua ibérica en la Galia Narbonense”, APL 12, 99-116.

Untermann 1980: J. Untermann, Trümmersprachen zwischen Grammatik und Geschichte, Opladen 1980.

Untermann 1983: J. Untermann, "Indogermanische Restsprachen als Gegenstand der Indogermanistik", en E. Vineis (ed.), Le lingue indoeuropee di frammentaria atestazione / Die indogermanischen Restsprachen (Atti del Convegno della Società Italiana di Glottologia e della Indogermanische Geschellschaft, Udine, 22-24 sett. 1981), Pisa 1983, 11-28.

Untermann 2000: J. Untermann, Wörterbuch des Oskisch-Umbrischen, Heidelberg 2000.

Untermann 2018: J. Untermann, Die vorrömische einheimische Toponymie des antiken Hispanien. Monumenta Linguarum Hispanicarum. Band VI, Wiesbaden 2018.

Vallejo 2013: J. M. Vallejo, "Hacia una definición del lusitano", PalHisp 13, 2013, 273-291.

Velaza 1991: J. Velaza, Léxico de inscripciones ibéricas (1976-1989), Barcelona 1991.

Vetter 1923: E. Vetter, “Etruskische Wortdeutungen”, Glotta 13/1, 1923, 138-149.

Vetter 1937: E. Vetter, Etruskische Wortdeutungen, Viena 1937.

Vetter 1953: E. Vetter, Handbuch der italischen Dialekte, Heidelberg 1953.

Villar 1995a: F. Villar, Estudios de celtibérico y de toponimia prerromana, Salamanca 1995.

Villar 1995b: F: Villar, A new Interpretation of Celtiberian Grammar, Innsbruck 1995.

Villar 1997: F. Villar, “The Celtiberian Language”, ZCPh 49-50, 1997, 898-949.

Villar 2007: F. Villar, “El Celta de la Península Ibérica”, en: P. Y. Lambert y G. J. Pinault (eds.), Gaulois et celtique continental, Ginebra 2007, 413-446.

Vineis 1983: E. Vineis (ed.), Le lingue indoeuropee di frammentaria atestazione / Die indogermanischen Restsprachen (Atti del Convegno della Società Italiana di Glottologia e della Indogermanische Geschellschaft, Udine, 22-24 sett. 1981), Pisa 1983.

Von Planta 1892-1897: R. von Planta, Grammatik der oskisch-umbrischen Dialekte, Estrasburgo 1892-1897.

Weisgerber 1931: L. Weisgerber, “Die Sprache der Festlandkelten”, Römisch-Germanische Kommission 20, 1931, 147-226 [= Rhenania Germano-Celtica, Bonn 1969, 11-85].

Whatmough 1970: J. Whatmough, The Dialects of Ancient Gaul. Prolegomena and Records of the Dialects, Cambridge, Mass. 1970 [con un índice de X. Delamarre en Veleia 21, 2004, 221-287]. 